\title{
Passivity Based Iterative Learning Control Design in the Discrete Repetitive Process Setting
}

\author{
Pavel Pakshin, Julia Emelianova, Milhail Emelianov, Krzysztof Galkowski and Eric Rogers
}

\begin{abstract}
Repetitive processes are important class of 2D systems with engineering applications and as a setting for iterative learning control (ILC) design. The application area for ILC is systems that execute the same finite duration task over and over again, with resetting to the starting location one each execution is complete. Previous research for linear dynamics has used the stability theory of linear repetitive processes to design control laws that have been experimentally verified. This paper applies the recently developed passivity theory for discrete repetitive process to ILC design. Based on this theory, a parametric description of a class of stabilizing controllers is obtained and a new design is developed that enhances the convergence properties of the implemented control law. An example using the model of the Quanser ${ }^{\circledR}$ flexible link is given to demonstrate the application of the new design.
\end{abstract}

\section{INTRODUCTION}

Repetitive processes [1], [2] have been the subject of considerable interest both in the design of stabilizing control laws and also their application to physical examples. The unique characteristic of these processes is a series of sweeps, termed passes, through a set of dynamics defined over a fixed finite duration known as the pass length. On each pass an output, termed the pass profile, is produced which acts as a forcing function on, and hence contributes to, the dynamics of the next pass profile. This, in turn, leads to the unique control problem where the output sequence of pass profiles generated can contain oscillations that increase in amplitude in the pass-to-pass direction.

Background on repetitive processes, including their use in modeling physical examples, can be found in [2] and the relevant cited references. These processes evolve over a subset of the upper-right quadrant of the $2 \mathrm{D}$ plane and are one form of 2D systems. As standard control action cannot prevent the increase in oscillations in the sequence of pass profiles, a stability theory for these processes has been developed [1], [2] using an abstract model of the dynamics

This work is partially supported by Russian Foundation for Basic Research, 16-08-00916a and by National Science Centre in Poland, grant No. 2015/17/B/ST7/03703.

Pavel Pakshin is with the Arzamas Polytechnic Institute of R.E. Alekseev Nizhny Novgorod State Technical University, 19, Kalinina Street, Arzamas, 607227, Russia and Lobachevsky State University of Nizhny Novgorod, Prospekt Gagarina, 23, 603950, Nizhny Novgorod, Russia pakshinpv@gmail.com

Julia Emelianova and Mikhail Emelianov are with Arzamas Polytechnic Institute of R.E. Alekseev Nizhny Novgorod State Technical University ,19, Kalinina Street, Arzamas, 607227, ttEmelianovaJulia@gmail.com

Krzysztof Galkowski is with the Institute of Control and Computation Engineering, University of Zielona Gora, Podgorna 50, 65-246 Zielona Gora, Poland K.Galkowski@issi.uz.zgora.pl

Eric Rogers is with the Department of Electronics and Computer Science, University of Southampton, Southampton SO17 1BJ, UK etardecs.soton.ac.uk in a Banach space setting. This abstract model includes all processes with linear dynamics and a constant pass length as special cases.

The stability theory for linear repetitive processes guarantees that a bounded, in the sense of the norm on the underlying function space, initial pass profile produces a bounded sequence of pass profiles. Two cases exist, either this property is required over the finite and fixed pass length or uniformly, i.e., independent of the pass length. The first of these properties is termed asymptotic stability and the second stability along the pass. Moreover, stability along the pass can be analyzed by considering the case when the pass length tends to infinity. Also the results of applying this theory to many special cases have been reported and also extended to control law design algorithms.

Mathematical models in the form of repetitive processes arise naturally in iterative learning control (ILC). This form of control is applied to systems that execute the same finite operation repeatedly, where each execution is termed a pass in this paper and the associate duration is termed the pass length (trial and iteration are also used in some of the literature). This form of control based on the idea that the performance of such systems can be improved by using information from the previous pass (or a finite number thereof), i.e., improve performance from pass-to-pass.

A common form of ILC constructs the current pass input as the sum of the input on the previous pass plus a correction term designed using previous pass information. This design approach was first introduced in [3] and ILC remains a very active area of research. A notable feature is the number of applications where experimental verification is also available and the survey papers [4], [5] are one starting points for the literature. The novel feature of ILC is that it allows the use of temporal information that would be non-causal for standard systems. This is because once a pass is complete, the complete data from the previous pass is available and hence non-causal temporal information cane be used in the control law with the proviso that such information has been generated on the previous pass (or passes).

Some applications areas for ILC will require the use of a nonlinear model and hence a stability theory for nonlinear repetitive processes is required. Recent years have seen the emergence of results on such a theory for nonlinear $2 \mathrm{D}$ systems. For example, the stability of nonlinear 2D systems written in the Roesser or Fornasini-Marchesini state-space model form have been considered in, e.g., [6]. In [7] a stability theory was developed for discrete nonlinear repetitive processes where vector Lyapunov functions were used to 
characterize the practically motivated stability properties.

In the case of standard, termed $1 \mathrm{D}$ in the multidimensional systems literature, nonlinear systems, dissipativity theory [8] is one of the most powerful methods for control design, where a particular form, known as passivity (and its generalizations) see, e.g., [9], [10], can be used to solve the global feedback stabilization problem for a wide class of systems. In [7] new results on a dissipativity approach to the stabilization of discrete and differential nonlinear repetitive processes were obtained by using a vector storage function approach that is different from that in, e.g., [11] and the resulting design based on divergence properties of this function.

This paper develops results on the use of this approach to ILC design for linear dynamics, where the analysis is for the discrete case and the extension to differential dynamics noted as a natural extension. The main result is a new design that has the potential of delivering improved performance over existing alternatives. An example based on the model of the Quanser ${ }^{\circledR}$ flexible link is used to illustrate the new design. Finally, areas for possible future research are briefly discussed.

\section{PROBLEM FORMULATION}

Let the nonnegative integer $k$ denote the pass number. Also let $u_{k}(p) \in \mathbb{R}^{l}, x_{k}(p) \in \mathbb{R}^{n}$ and $y_{k}(p) \in \mathbb{R}^{m}$ be the input, state and output vectors, respectively, at instant $0 \leq p \leq$ $T-1<\infty$. Then in the ILC setting the dynamics of the linear uncontrolled system are described by

$$
\begin{aligned}
x_{k}(p+1) & =A x_{k}(p)+B u_{k}(p), \\
y_{k}(p) & =C x_{k}(p), k=0,1, \ldots
\end{aligned}
$$

with assumed boundary conditions

$$
y_{0}(p)=C x_{0}(p), 0 \leq p \leq T-1, x_{k}(0)=x_{0}, k \geq 0
$$

Suppose that $y_{\text {ref }}(p)$ denotes the supplied reference vector over $0 \leq p \leq T-1$. Then $e_{k}(p)=y_{\text {ref }}(p)-y_{k}(p)$ is the error on pass $k$ and the problem is to construct a sequence of input functions such that the performance achieved is gradually improving with each successive pass can be expressed as a convergence condition on the input and error, i.e.,

$$
\lim _{k \rightarrow \infty}\left\|e_{k}(p)\right\|=0, \lim _{k \rightarrow \infty}\left\|u_{k}(p)-u_{\infty}(p)\right\|=0 .
$$

The paper extends the set of ILC laws based on a passivity approach, for which the next section gives the required background.

This paper paper considers discrete dynamics but results given extend in a natural manner to linear differential dynamics with state-space model

$$
\begin{aligned}
& \dot{x}_{k+1}(t)=A x_{k+1}(t)+B u_{k+1}(t), \\
& y_{k+1}(t)=C x_{k+1}(t), x_{k}(0)=x_{0} .
\end{aligned}
$$

where $t$ denotes the along the trial variable and the remaining notation is as in the discrete case.

\section{PRELIMINARIES}

This section summarizes the required results from the existing passivity based stability theory, which follows in the main [7] for nonlinear dynamics

\section{A. General stability result}

The state-space model of the discrete nonlinear repetitive processes considered is

$$
\begin{aligned}
x_{k+1}(p+1) & =f_{1}\left(x_{k+1}(p), y_{k}(p), u_{k+1}(p)\right), \\
y_{k+1}(p) & =f_{2}\left(x_{k+1}(p), y_{k}(p), u_{k+1}(p)\right),
\end{aligned}
$$

where on pass $k x_{k}(p) \in \mathbb{R}^{n_{x}}$ is the current pass state vector, $y_{k}(p) \in \mathbb{R}^{n_{y}}$ is the pass profile vector, $u_{k}(p) \in \mathbb{R}^{n_{u}}$ is the control input vector and $f_{1}$, and $f_{2}$ are nonlinear functions. Also it is assumed that $f_{1}(0,0,0)=0, f_{2}(0,0,0)=0$ and hence an equilibrium at the origin.

The boundary conditions, i.e., the pass state initial vector sequence and the initial pass profile, are assumed to be known and have the form

$$
\begin{aligned}
x_{k+1}(0) & =d_{k+1}, k \geq 0, \\
y_{0}(p) & =f(p), 0 \leq p \leq T-1,
\end{aligned}
$$

where $d_{k+1} \in \mathbb{R}^{n_{x}}$ is a known vector sequence, $f(p) \in \mathbb{R}^{n_{y}}$ denotes a vector whose entries are known functions of $p$, $0 \leq p \leq T-1$. Moreover, if $\|q\|$ denotes the norm of a vector $q$, it is assumed that $f(p)$ and $d_{k+1}$ satisfy

$$
\|f(p)\|^{2} \leq M_{f},\left\|d_{k+1}\right\|^{2} \leq \kappa_{d} \zeta_{d}^{k}, k=0,1, \ldots
$$

where $M_{f}>0$ is a finite scalar, $\kappa_{d}>0$ is independent of $T$ and $0<\zeta_{d}<1$ determines the rate of convergence of the pass state initial vector sequence. Throughout this paper, it is assumed that the boundary conditions considered satisfy (7) and no further explicit mention of these conditions is made.

The unique control problem for repetitive processes is the possible presence of oscillations that increase in amplitude from pass-to-pass. Hence the stability theory for repetitive processes demands that a bounded initial pass profile produces a bounded sequence of pass profiles. The strongest requirement is for this property to hold for all possible values of the pass length, which can be analyzed mathematically by considering $T \rightarrow \infty$.

This paper uses the following definition of stability for discrete nonlinear repetitive processes.

Definition 1: A nonlinear repetitive process described by (5) and (6) is said to be exponentially stable if there exist $\kappa>0$ and $0<\lambda<1$ such that

$$
\left\|x_{k}(p)\right\|^{2}+\left\|y_{k}(p)\right\|^{2} \leq \kappa \lambda^{k+p},
$$

where $\kappa$ is independent of $T$, i.e., of the pass length. In contrast to the $1 \mathrm{D}$ case , the full increment of a candidate Lyapunov function for the case of (5) cannot be used to apply the second Lyapunov method for stability analysis as in the standard systems case.

This fact has stimulated research using a vector Lyapunov function and the discrete counterpart of the divergence operator (referred to as the divergence operator in the rest of 
this paper) instead of the full increment. The analysis below employs a vector Lyapunov function of the following form for discrete processes

$$
V(x, y)=\left[\begin{array}{c}
V_{1}\left(x_{k+1}(p)\right) \\
V_{2}\left(y_{k}(p)\right)
\end{array}\right],
$$

where $V_{1}(x)>0, x \neq 0, \quad V_{2}(y)>0, y \neq 0, \quad V_{1}(0)=$ $0, V_{2}(0)=0$. The divergence operator of this function along the trajectories of (5) is defined as

$$
\begin{aligned}
\mathcal{D}_{d} V\left(x_{k+1}(p),\right. & \left.y_{k}(p)\right) \\
& =\Delta_{p} V_{1}\left(x_{k+1}(p)\right)+\Delta_{k} V_{2}\left(y_{k}(p)\right),
\end{aligned}
$$

where

$$
\begin{aligned}
\Delta_{p} V_{1}\left(x_{k+1}(p)\right) & =V_{1}\left(x_{k+1}(p+1)\right)-V_{1}\left(x_{k+1}(p)\right) \\
\Delta_{k} V_{2}\left(y_{k}(p)\right) & =V_{2}\left(y_{k+1}(p)\right)-V_{2}\left(y_{k}(p)\right)
\end{aligned}
$$

The following theorem holds.

Theorem 1: [7] Consider a nonlinear discrete repetitive process described by (5) and (6). Suppose also that there exist positive constants $c_{1}, c_{2}, c_{3}$ such that the vector Lyapunov function $V$ and its divergence along the trajectories of (5) satisfy the inequalities

$$
\begin{array}{r}
c_{1}\left\|x_{k+1}(p)\right\|^{2} \leq V_{1}\left(x_{k+1}(p)\right) \leq c_{2}\left\|x_{k+1}(p)\right\|^{2}, \\
c_{1}\left\|y_{k}(p)\right\|^{2} \leq V_{2}\left(y_{k}(p)\right) \leq c_{2}\left\|y_{k}(p)\right\|^{2}, \\
\mathcal{D}_{d} V\left(x_{k+1}(p), y_{k}(p)\right) \leq-c_{3}\left(\left\|x_{k+1}(p)\right\|^{2}+\left\|y_{k}(p)\right\|^{2}\right) .
\end{array}
$$

Then this process is exponentially stable.

\section{B. General passivity result}

A powerful method in the analysis and control of $1 \mathrm{D}$ systems is dissipativity theory [8], especially the particular case of passivity theory [8], [9] where an extension of a Lyapunov function termed a storage function is used. This section considers design for discrete linear repetitive processes in this setting.

Introduce, for analysis and control law design purposes only as discussed below, the auxiliary vector $z_{k}(p) \in \mathbb{R}^{n_{u}}$ given by

$$
z_{k+1}(p)=g\left(x_{k+1}(p), y_{k}(p), u_{k+1}(p)\right),
$$

where $g$ is a nonlinear function such that $g(0,0,0)=0$ and define the dissipativity property as follows.

Definition 2: A discrete nonlinear repetitive process described by (5) and (6) is said to be exponentially dissipative if there exists a vector function (9), a scalar function $S(u, z)$ and positive scalars $c_{1}, c_{2}$ and $c_{3}$ such that

$$
\begin{aligned}
c_{1}\|x\|^{2} & \leq V_{1}(x) \leq c_{2}\|x\|^{2}, \\
c_{1}\|y\|^{2} & \leq V_{2}(y) \leq c_{2}\|y\|^{2}, \\
\mathcal{D}_{d} V\left(x_{k+1}(p), y_{k}(p)\right) & \leq S\left(u_{k+1}(p), z_{k+1}(p)\right) \\
& -c_{3}\left(x_{k+1}(p)+y_{k}(p)\right) .
\end{aligned}
$$

In the particular case when $S(u, z)=z^{T} G u$, where $G$ is a constant square matrix of compatible dimensions, a repetitive process described by (5) and (6) is said to be exponentially $G$-passive, see [12] for the 1D systems case. Since (5) does not involve full increments, as in the case of ordinary 1D difference equations, it is impossible to use cross terms in the vector storage function (9).

The auxiliary vector $z$ of (11) can be used to achieve certain dissipativity properties and for the case of passivity this is known as passivation [13]. Moreover, the choice of this vector depends on the choice of storage function $V$ and it is a separate complex problem (similar to the choice of a Lyapunov function for a nonlinear systems). The problem is to find a pair $(z, V)$ satisfying the definition of passivity and later it will be shown how this pair and corresponding feedback law can be chosen for considered special cases.

The following theorem allows the application of the passivity property to stabilizing control design.

Theorem 2: [7] Suppose that a discrete nonlinear repetitive process described by (5), (6) and (11) is exponentially $G$ passive. Suppose also that there exists a function $\varphi(z)$ such that $\varphi(0)=0$ and $z^{T} G \varphi(z)>0$ if $z \neq 0$. Then the control law

$$
u_{k+1}(p)=-\varphi\left(z_{k+1}(p)\right)
$$

results in controlled dynamics that are exponentially stable.

Application of this theory requires the selection of a suitable $V_{1}\left(x_{k+1}(p)\right)$ and $V_{2}\left(y_{k}(p)\right)$ and in the nonlinear case there is, as for other nonlinear systems, no general method for selecting these functions. In the particular case of linear dynamics, it is possible to chose these functions as quadratic and the rest of this paper develops a passive control law design for discrete linear repetitive processes, with a particular application to ILC design.

\section{A particular example of the general approach}

Consider the 1D discrete linear system with state equation

$$
x(q+1)=A x(q)+B u(q), q=0,1, \ldots,
$$

where $x \in \mathbb{R}^{n_{x}}$ is state vector, $u \in \mathbb{R}^{n_{u}}$ is control input vector, $A$ and $B$ are constant matrices of compatible dimensions. Assume that matrix $A$ is stable and define the matrix $P \succ 0$ (i.e., a symmetric positive definite matrix) as a solution of the Lyapunov inequality

$$
A^{T} P A-P+Q \prec 0,
$$

where $Q \succ 0$. Consider also a storage function for (13) of the form of $V(x)=x^{T} P x$. The full increment of this function along the trajectories of (13) is

$$
\begin{aligned}
\Delta V(x(q)) & =x(q)^{T}\left(A^{T} P A-P\right) x(q) \\
& +\left(2 x(q)^{T} A^{T} P B+u(q)^{T} B^{T} P B\right) u(q) .
\end{aligned}
$$

Using [10], (13) is passive with respect to input $u(q)$ and output $y(q)=2 B^{T} P A x(q)+B^{T} P B u(q)$ and the control law

$$
u(q)=-\left(I+\frac{1}{2} G(x(q))^{-1} G(x(q)) B^{T} P A x(q),\right.
$$

where $G(x)$ is a matrix function that is positive semi-definite for all $x \in \mathbb{R}^{n_{x}}$, stabilizes system (13). Moreover, the matrices $Q$ and $G(x)$ define a set of stabilizing controllers 
of the form (16) and by varying these matrices it is possible to alter the dynamical properties of the controlled system.

If matrix $A$ is unstable define the pair of matrices $P \succ 0$ and $K$ as a solution to

$$
(A+B K)^{T} P(A+B K)-P+Q \prec 0,
$$

and apply the results above to the system

$$
x(q+1)=\bar{A} x(q)+B v(q),
$$

where $\bar{A}=A+B K$ is a stable matrix and $v(q)=u(q)-$ $K x(q)$. In this case the set of stabilizing controls are given by

$$
u(q)=\left[K-\left(I+\frac{1}{2} G(x(q))\right)^{-1} G(x(q)) B^{T} P \bar{A}\right] x(q)
$$

and again by varying $Q$ and $G(x)$ the dynamical properties of the controlled system can be modified. The analysis in the remainder of this paper aims to transfer this design freedom to ILC design for linear dynamics.

\section{PASSIVITY-BASED ILC DESIGN}

A commonly used ILC law is to select the input on the current pass as that used on the previous pass plus a correction. In this paper the ILC law on pass $k+1$ is of the form

$$
u_{k+1}(p)=u_{k}(p)+\Delta u_{k+1}(p)
$$

where $\Delta u_{k+1}(p)$ is the correction term to be designed. The novel feature of ILC is all information generated on a completed pass is available for use in the computation of $\Delta u_{k+1}(p)$. This allows the use of temporal information that is non-causal in the standard sense provided it is generated on a previous pass. In this section discrete linear dynamics are considered.

To write the ILC dynamics as a discrete linear repetitive process, introduce, for analysis purposes only, the vector

$$
\xi_{k+1}(p+1)=x_{k+1}(p)-x_{k}(p) .
$$

Then the controlled dynamics can be written as

$$
\begin{aligned}
\xi_{k+1}(p+1) & =A \xi_{k+1}(p)+B \Delta u_{k+1}(p-1), \\
e_{k+1}(p) & =-C A \eta_{k+1}(p)+e_{k}(p) \\
& -C B \Delta u_{k+1}(p-1) .
\end{aligned}
$$

If the control correction term provides exponential stability of (22) then the ILC law (20) converges in the sense that conditions (3) hold.

Introduce the notation $A_{11}=A, \quad A_{11 c}=A+$ $B K_{1}, A_{12}=0, A_{12 c}=B K_{2}, A_{21}=-C A, A_{21 c}=$ $-C A-C B K_{1}, A_{22}=I, A_{22 c}=I-C B K_{2}, B_{1}=$ $B, B_{2}=-C B, v_{k+1}(p)=\Delta u_{k+1}(p-1)-K_{1} x_{k+1}(p)-$ $K_{2} y_{k}(p)$ and rewrite (22), for technical purposes only, in the following equivalent form

$$
\begin{array}{r}
x_{k+1}(p+1)=A_{11 c} x_{k+1}(p)+A_{12 c} y_{k}(p)+B_{1} v_{k+1}(p), \\
y_{k+1}(p)=A_{21 c} x_{k+1}(p)+A_{22 c} y_{k}(p)+B_{2} v_{k+1}(p), \\
0 \leq p \leq T-1 ; k=0,1,2, \ldots
\end{array}
$$

and to simply the notation $\bar{x}_{k+1}(p)=\left[\begin{array}{c}\xi_{k+1}(p) \\ e_{k}(p)\end{array}\right], \bar{A}=$ $\left[\begin{array}{ll}A_{11} & A_{12} \\ A_{21} & A_{22}\end{array}\right]$ $\bar{B}=\left[\begin{array}{l}B_{1} \\ B_{2}\end{array}\right], K=\left[\begin{array}{ll}K_{1} & K_{2}\end{array}\right]$.

Assume that matrix $K$ satisfies the following matrix inequality

$$
(\bar{A}+\bar{B} K)^{T} P(\bar{A}+\bar{B} K)+Q+K^{T} R K \preceq 0
$$

where $Q \succ 0$ and $R \succ 0$ are weighting matrices. This inequality is easily reduced to a Linear Matrix Inequality (LMI) with respect to $X=\operatorname{diag}\left[X_{1} X_{2}\right]$, where $X_{1}=P_{1}^{-1}$ and $X_{2}=P_{2}^{-1}$ and $Y=K X$ :

$$
\left[\begin{array}{cccc}
X & (\bar{A} X+\bar{B} Y)^{T} & X & Y^{T} \\
\bar{A} X+\bar{B} Y & X & 0 & 0 \\
X & 0 & Q^{-1} & 0 \\
Y & 0 & 0 & R^{-1}
\end{array}\right] \succeq 0
$$

If these inequalities are feasible then $K=Y X^{-1}$.

The next theorem describes a set of stabilizing control law for (22).

Theorem 3: The dynamics of (22) are exponentially passive with respect to the output

$$
z_{k+1}(p)=2 \bar{B}^{T} P \bar{A}_{c} \bar{x}_{k+1}(p)+\bar{B}^{T} P \bar{B} v_{k+1}(p),
$$

where

$$
\bar{A}_{c}=\bar{A}+\bar{B} K
$$

and a set of feedback stabilizing control laws for the system with this output is given by

$$
\Delta u_{k+1}(p-1)=F\left(\bar{x}_{k+1}(p)\right) \bar{x}_{k+1}(p),
$$

where

$$
\begin{aligned}
& F\left(\bar{x}_{k+1}(p)\right)=\left[I+G\left(\bar{x}_{k+1}(p)\right) \bar{B}^{T} P \bar{B}\right]^{-1}[(I \\
& \left.\left.-G\left(\bar{x}_{k+1}(p)\right) \bar{B}^{T} P \bar{B}\right) K-2 G\left(\bar{x}_{k+1}(p)\right) \bar{B}^{T} P \bar{A}\right],
\end{aligned}
$$

where $G(\bar{x})$ is an arbitrary matrix of compatible dimensions that is positive semi-definite for all $\bar{x} \in \mathbb{R}^{n_{x}+n_{y}}$. and the pair of matrices $P=X^{-1}$ and $K$ solve (25).

Proof: Consider the candidate vector storage function as (9) with $V_{1}\left(\xi_{k+1}(p)\right)=\xi_{k+1}^{T}(p) P_{1} \xi_{k+1}(p), V_{2}\left(e_{k}\right)=$ $e_{k}^{T}(t) P_{2} e_{k}(t)$, where $P_{1} \succ 0$ and $P_{2} \succ 0$. Calculating divergence of (9) along the trajectories of (23) gives

$$
\begin{aligned}
\mathcal{D}_{d} V=\bar{x}^{T}\left(\bar{A}_{c}^{T} P \bar{A}_{c}-P\right) \bar{x}+\left(2 \bar{x}^{T} \bar{A}_{c}^{T} P \bar{B} v\right. \\
\left.+v^{T} \bar{B}^{T} P \bar{B}\right) v
\end{aligned}
$$

where $P=\operatorname{diag}\left[P_{1} P_{2}\right]$. Choose the output $z$ as (26), then

$$
\mathcal{D}_{d} V(x, y) \leq-\bar{x}^{T} Q \bar{x}+z^{T} v
$$

and it follows from (30) that (23) is exponentially passive with respect to input $v$ and output (26). By Theorem 2, the control law

$$
v_{k+1}(p)=-G\left(\bar{x}_{k+1}(p)\right) z_{k+1}(p)
$$




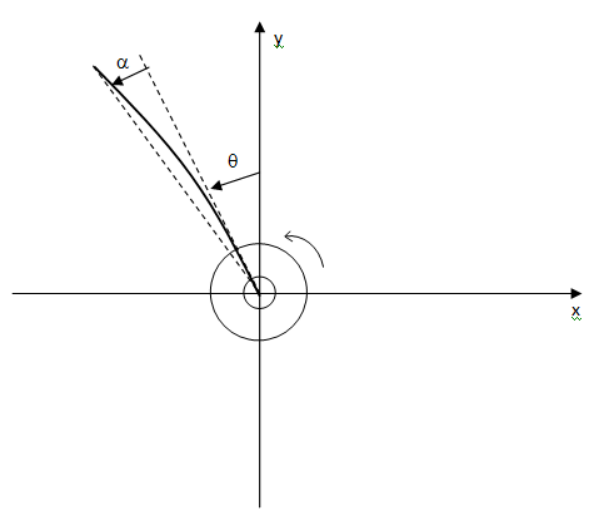

Fig. 1. Angles of the rotary flexible link.

where $G \bar{x}$ is defined above results in exponential stability of (23). Then by routine calculations it follows from (31), (26) and definition of $v$ that each control law from (27) results in exponential stability of (22).

\section{EXAMPLE}

As the example, ILC design for the Quanser ${ }^{\circledR}$ flexible link is considered. The mathematical model of the dynamics of the link in the horizontal position is easily obtained from Lagrange's equations of motion, see Quanser ${ }^{\circledR}$ lab manual for details [14]. The state-space form of this model is as follows.

$$
\dot{x}=A_{o} x+B_{o} u,
$$

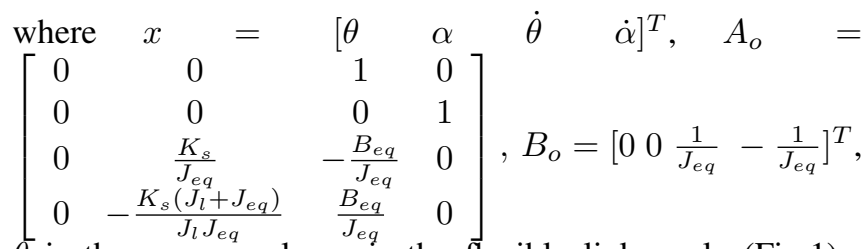
$\theta$ is the servo angle, $\alpha$ is the flexible link angle (Fig.1), $K_{s}$ is the stiffness of the flexible link, $J_{e q}$ is the moment of inertia of the servo, $B_{e q}$ is the viscous friction coefficient of the servo, $J_{l}$ is the moment of inertia of the flexible link.

The required reference trajectory is the required servo angle $\theta(t)$, which is chosen to simulate a "pick and place" process of duration 2 sec's and is shown in Fig. 2.

For the flexible link model (32) $A=\exp \left(A_{o} T_{s}\right), B=$ $\int_{0}^{T_{s}} \exp \left(A_{o} \tau\right) d \tau$, where $T_{s}$ is sampling time, and $C=$ $\left[\begin{array}{llll}1 & 0 & 0 & 0\end{array}\right]$. Since $C_{d} B_{d} \neq 0$ it is possible to use the model (22) for ILC design and by Theorem 3 the set of stabilizing control laws is given by

$$
\begin{aligned}
\Delta u_{k+1}(p-1)= & {\left[I+G \bar{B}^{T} P \bar{B}\right]^{-1}\left[\left(I-G \bar{B}_{d}^{T} P \bar{B}\right) K\right.} \\
& \left.-2 G^{T} \bar{B}^{T} P \bar{A}\right]\left[\xi_{k+1}^{T}(t) e_{k}^{T}(p)\right]^{T},
\end{aligned}
$$

where $\bar{A}=\left[\begin{array}{cc}A & 0 \\ -C A & I\end{array}\right], \bar{B}_{d}=\left[\begin{array}{c}B \\ -C B_{d}\end{array}\right]$ and matrices $K$ and $P$ are given by the solution of the LMI (25). Only the case of linear feedback is considered ( $G$ does not depend on $\bar{x}$ in this case)

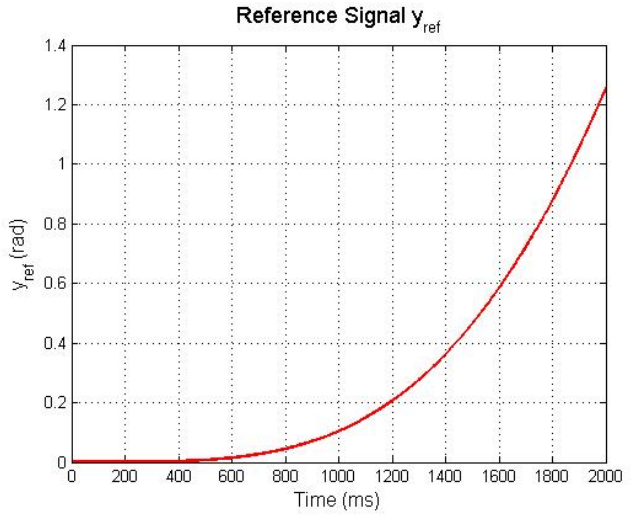

Fig. 2. The reference trajectory

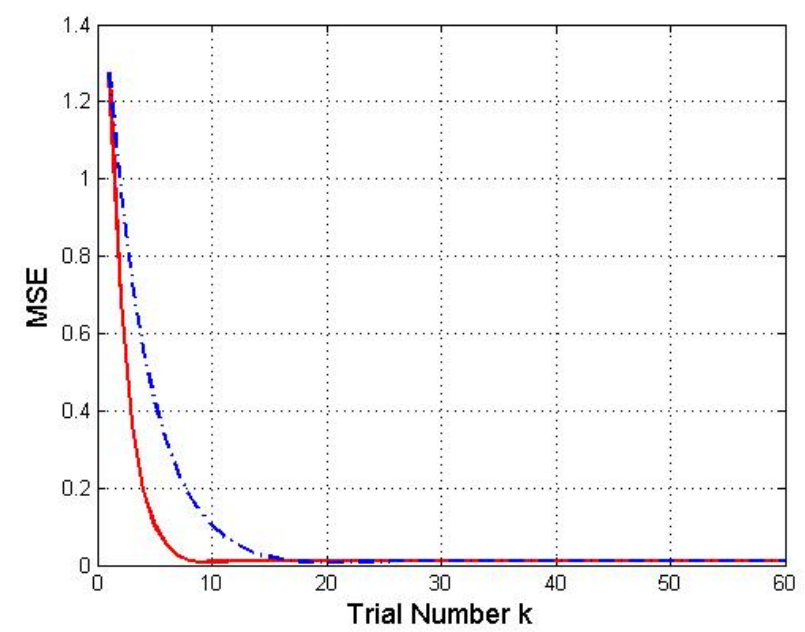

Fig. 3. Comparing the performance of the controllers based on using of discrete model: $G=0$ (dashed line), $G=1$ (solid line).

Choosing $T_{s}=0.01 \mathrm{~s}$. and applying Theorem 3 for the case with $G=0$ and

$$
Q_{1}=\operatorname{diag}\left[10^{-5} 10^{-5} 200.5\right], \quad Q_{2}=10^{5}, \quad R=10^{-9}
$$

gives

$$
F=\left[\begin{array}{lllll}
-27.4 & -1.29 & -0.34 & -0.005 & 6.56
\end{array}\right] .
$$

Applying Theorem 3 with $G=1$ and with the same $\Delta, Q_{1}, Q_{2}$ and $R$ gives

$$
F=\left[\begin{array}{lllll}
-16.83 & -1.24 & -0.29 & -0.0064 & 7.77
\end{array}\right] .
$$

The choice of $Q$ and $R$ is based on both linear quadratic regular theory reasons and engineering reasons. In particular, the choice above reflects the premise that in applications the learning error and derivative of the servo angle are key variables in ILC design.

To compare designs

$$
E(k)=\sqrt{\frac{1}{T} \sum_{p=0}^{T}\left\|e_{k}(p)\right\|^{2}},
$$


which is the mean square error computed along trial $k$. and Fig 3) gives the results from application of the two control laws. These confirm that monotonic trial-to-trial error convergence occurs for both designs but the choice $G=10$ has better performance in terms of the convergence rate. In particular, the initial error is reduced by a factor of 10 in 5 trials for $G=10$ but 10 trials are required under $G=0$. Moreover, convergence occurs after 10 trials for $G=10$ but the stability along the trial with $G=0$ requires 20 trials. Further tuning of this ILC is possible by varying the matrices $Q$ and $G(x)$ and hence the ILC design performance can be tailored to the requirements of the example under consideration.

\section{CONCLUSIONS}

This paper has shown how passivity based designs for nonlinear repetitive processes can be applied to the design of ILC laws, where in this paper attention has focused on discrete linear dynamics with a straightforward extension to differential linear dynamics. In the resulting design, the parameter $G$ can vary depending on the number of trials. This provides the possibility of trial-dependent adaptation in passto-pass error convergence. The full implications of this result is the subject of ongoing research. In principle, the passivity setting extends to ILC design for nonlinear dynamics and this topic is also under investigation.

\section{REFERENCES}

[1] E. Rogers and D. H. Owens, Stability Analysis for Linear Repetitive Processes. Lecture Notes in Control and Information Sciences, Vol.175. Berlin: Springer-Verlag, 1992.
[2] E. Rogers, K. Gałkowski, and D. Owens, Control Systems Theory and Applications for Linear Repetitive Processes. Lecture Notes in Control and Information Sciences, Vol. 349. Berlin: Springer-Verlag, 2007.

[3] S. Arimoto, S. Kawamura, and F. Miyazaki, "Bettering operation of robots by learning," Journal of Robotic Systems, vol. 1, no. 2, pp. 123-140, 1984.

[4] H.-S. Ahn, Y.-Q. Chen, and K. L. Moore, "Iterative learning control: Brief survey and categorization," IEEE Transactions on Systems, Man and Cybernetics, Part C: Applications and Reviews, vol. 37, no. 6, pp. 1099-1121, 2007.

[5] D. Bristow, M. Tharayil, and A. Alleyne, "A survey of iterative learning control," IEEE Control Systems Magazine, vol. 26, no. 3, pp. 96-114, 2006.

[6] N. Yeganefar, N. Yeganefar, M. Ghamgui, and E. Moulay, "Lyapunov theory for 2-D nonlinear Roesser models: Application to asymptotic and exponential stability," IEEE Transactions on Automatic Control, vol. 58, pp. 1299-1304, 2013.

[7] P. V. Pakshin, J. Emelianova, K. Gałkowski, and E. Rogers, "Dissipivity and stabilization of nonlinear repetitive processes," Systems \& Control Letters, vol. 91, pp. 14-20, 2016.

[8] J.C.Willems, "Dissipative dynamical systems part I: General theory," Arch. Rational Mech. Analysis, vol. 45, pp. 325 - 351, 1972.

[9] C. Byrnes, A. Isidori, and J. Willems, "Passivity, feedback equivalence and the global stabilization of minimun phase nonlinear systems," IEEE Trans. Automat. Contr, vol. 36, pp. 1228 - 1240, 1991.

[10] W. Lin and C. Byrnes, "Passivity and absolute stabilization of a class of discrete-time nonlinear systems," Automatica, vol. 31, pp. 263 267, 1995.

[11] W. Haddad, V. Chellabona, and S. Nersesov, "Vector dissipativity theory and stability of feedback interconnections for large-scale nonlinear dynamical systems," Int.J. Control, vol. 77, pp. 907-919, 2004.

[12] A. Fradkov and D. Hill, "Exponential feedback passivity and stabilizability of nonlinear systems," Automatica, vol. 34, pp. 697 - 703, 1998.

[13] H. Khalil, Nonlinear Systems. Third Edition. New Jersey: Prentice Hall, 2002.

[14] J. Apkarian, P. Karam, and M. Lévis, Workbook on Flexible Link Experiment for Matlab ${ }^{\circledR} /$ Simulink ${ }^{\circledR}$ Users. Quanser, 2011. 